\title{
New ascending aortic aneurysm model in rats reproduces main structural features of degenerative ascending thoracic aortic aneurysms in human beings
}

\author{
Narcis Costin Radu, MD, ${ }^{\mathrm{a}, \mathrm{b}}$ Marianne Gervais, $\mathrm{PhD},{ }^{\mathrm{a}}$ Stéphanie Michineau, $\mathrm{PhD},{ }^{\mathrm{a}}$ Raphaël Blanc, $\mathrm{MD},{ }^{\mathrm{a}, \mathrm{c}}$ \\ Alexandre Fifre, $\mathrm{PhD},{ }^{\mathrm{a}}$ Ernst Wilhelm Matthias Kirsch, $\mathrm{MD}, \mathrm{PhD},{ }^{\mathrm{a}, \mathrm{b}}$ and Eric Allaire, MD, $\mathrm{PhD}{ }^{\mathrm{a}, \mathrm{d}}$
}

\begin{abstract}
Objectives: The singularity of the ascending aorta regarding mechanisms driving aneurysm formation requires the development of specific animal models. We investigated if adventitial elastase application results in ascending aorta aneurysms in rats.
\end{abstract}

\begin{abstract}
Methods: Adult Lewis rats $(\mathrm{n}=26)$ were anesthetized, their ascending aortas measured by transthoracic ultrasound, and exposed via median sternotomy. Elastase or saline was applied on the ascending aortic adventitia. Ascending aorta diameters were monitored by ultrasound at 10 and 30 days, when the animals were killed. Wall area was measured on orcein stained sections. Matrix metalloproteinase- 2 and matrix metalloproteinase- 9 levels were quantified on gelatin zymography.
\end{abstract}

Results: Following elastase application, ascending aortic diameter increased at 10 and 30 days follow-up by $38 \%$ and $44 \%$, respectively $(P=.004)$. Despite thinning of the media secondary to vascular dilation, standardized medial area was not different between elastase-treated aortas and controls. Standardized total wall area had a significant increase in treated aortas compared with controls. Active matrix metalloproteinase- 2 was significantly increased at 30 days in treated aortas, whereas active matrix metalloproteinase- 9 was no different from controls.

Conclusions: Elastase application on rat ascending aortic adventitia produced aneurysms, creating a reproducible model. Aortic wall remodeling evolved toward an increase in total wall area, reproducing the main structural features of this disease in human beings. (J Thorac Cardiovasc Surg 2013;145:1627-34)

Sixty percent of thoracic aortic aneurysms involve the ascending aorta and predispose patients to life-threatening complications, including aortic dissection and rupture. ${ }^{1}$ Most of these ascending thoracic aortic aneurysms (ATAAs) are degenerative; that is, independent of an overt connective-tissue disorder or a definite genetic background. Although the incidence and prevalence of thoracic aortic aneurysms are increasing, ${ }^{2}$ the mechanisms by which ATAAs develop in individuals with no identified genetic syndrome remain poorly understood. Recent research on arterial aneurysms has benefited from the development of animal models that have provided some insight into the

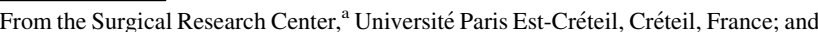
Department of Cardiovascular and Thoracic Surgery, ${ }^{\mathrm{b}}$ Department of Neuroradiology, ${ }^{\mathrm{c}}$ and Department of Vascular Surgery, ${ }^{\mathrm{d}}$ APHP Hôpital Henri Mondor, Créteil, France.

This study was supported by a grant from the French Académie Nationale de Médecine, by a grant from the Fondation de l'Avenir pour la Recherche Médicale, and by a grant from the European Union ("Fighting Aneurysmal Disease" collaborative project FP7 Health 2007-A).

Disclosures: Authors have nothing to disclose with regard to commercial support.

Received for publication May 15, 2012; revisions received July 3, 2012; accepted for publication July 26, 2012; available ahead of print Aug 29, 2012.

Address for reprints: Narcis Costin Radu, MD, Department of Cardiovascular and Thoracic Surgery, APHP Hôpital Henri Mondor, 51 Ave du Maréchal de Lattre de Tassigny, 94000 Créteil, France (E-mail: ncostin.radu@gmail.com). $0022-5223 / \$ 36.00$

Copyright (c) 2013 by The American Association for Thoracic Surgery http://dx.doi.org/10.1016/j.jtcvs.2012.07.050
}

mechanisms of aneurysmal disease, and have opened up research on innovative treatment options. ${ }^{3-6}$ There is no reproducible model for degenerative ATAAs located in a segment of the aorta with particular characteristics likely to determine mechanisms of formation and particular therapeutic approaches. Besides specific anatomic, histologic, mechanical, and hemodynamic characteristics, ${ }^{7}$ the ascending aorta originates from an embryonic structure distinct from the origin of other segments of the aorta. Vascular smooth muscle cells (VSMCs) of the abdominal aorta have mesenchymal origin, whereas VSMCs of the ascending aorta originate in the cardiac neural crest exhibiting lineage-dependant responses to growth factors. ${ }^{8,9}$ As an illustration of these specificities, risk factors and pathologic features of ATAAs differ from those of aneurysms in other locations along the aorta. ${ }^{10}$ These specificities prompted us to develop an animal model ATAA.

The goal of our study was to test the hypothesis that elastase application onto the ascending aorta in rats would produce aneurysms sharing common features of human ATAAs.

\section{MATERIALS AND METHODS \\ Animals}

Twenty-six adult, male, in-bred 16-week old Lewis rats (mean weight 445 g) (Janvier, Le Genest-St-Isle, France) were randomly assigned for 


\section{Abbreviations and Acronyms \\ $\alpha$-SMA $=$ alpha smooth muscle actin \\ ATAA $=$ ascending thoracic aortic aneurysm \\ MMP = matrix metalloproteinase \\ $\mathrm{VSMC}=$ vascular smooth muscle cell}

treatment and followed for 30 days. The animals were weighed, anesthetized by intraperitoneal injection of $5.5 \mathrm{mg} / 100 \mathrm{~g}$ body weight pentobarbital (Ceva Santé Animale, Libourne, France), and shaved. Animals were treated and cared for in accordance with The European Convention for the protection of vertebrate animals used for experimental and other scientific purposes of the European Council.

\section{Aneurysm Model}

The animals were anesthetized and an anterior midline incision was made from the neck to the xyphoid process. Rats were subjected to retrograde intubation and ventilated with room air using a Harvard Rodent Ventilator 683 (Harvard Apparatus, Holliston, Mass), set at a respiratory rate of 55 to 65 breaths per minute, with a tidal volume of $2 \mathrm{~mL}$. A median sternotomy was performed and the thymus was retracted toward the neck to expose the ascending aorta.

The ascending aorta was dissected free from the pulmonary artery and $100 \mu \mathrm{L}$ elastase solution $(160 \mathrm{U} / \mathrm{mL})$ (porcine pancreas elastase, Serva Electrophoresis, Heidelberg, Germany) or physiologic saline (Fresenius Kabi, Sèvres, France) was applied on a swab that was wrapped around the aorta during 40 minutes. At the end of treatment the thoracic wall was closed in 2 layers with separate stitches of 4-0 polypropylene (Prolene; Ethicon, Johnson and Johnson Co, Cornelia, Ga). Pneumothorax was reduced by obstructing outflow on the ventilator for 4 to 5 breaths just before tight closure of the chest. The respiratory rate was gradually decreased and the rat was extubated when it showed evidence of good spontaneous respiration. During follow-up, animals were monitored daily and given water and chow ad libitum.

\section{Ultrasound Imaging}

The noninvasive method for measuring diameter of the ascending aorta in rats by ultrasound has been described elsewhere. ${ }^{11}$ Aortic imaging was performed in anesthetized animals in dorsal recumbence, using a VINGMED CFM 750 scanner with a transducer at an operating frequency of $9 \mathrm{MHz}$ (GE, Fairfield, Conn). The transducer was placed on the left hemi thorax and oriented to provide a longitudinal image of the ascending aorta in parasternal incidence. Localization of the vessels was determined by pulse Doppler signal. Ascending aortic diameters were measured in late systole, strictly marking the inner aortic diameter at midpoint between the sinotubular junction and the origin of the brachiocephalic trunk. The mean of 3 separate measurements was retained. Ultrasound measurements were performed under the same conditions before surgical intervention, at 10 and 30 days of follow-up, by the same observer, blinded with respect to the animal's experiment group. Arterial blood pressure was not monitored.

\section{Tissue Harvesting and Histology}

Before euthanasia, ultrasound measurements were repeated under general anesthesia. First animals killed from the elastase-treated and control groups underwent angiography by direct exposure of the right carotid artery and injection of $3 \mathrm{~mL}$ contrast agent through a catheter (Insyte Autoguard 22 GA, 25 mm, BD, Franklin Lakes, NJ). After orotracheal intubation the thorax was opened under the xiphoid process and the anterior chest wall was removed. An incision was made in the right atrium to provide an outlet for blood and perfusate. The left ventricle was then punctured with a 21-G needle inserted in the direction of the left ventricular outflow tract. The needle was attached to a perfusion system that provided initial perfusion with normal saline at $100 \mathrm{~mm} \mathrm{Hg}$. The saline perfusion was continued until outflow from the right atrium incision was clear and the liver appeared exsanguinated. The animal was then perfused with $70 \%$ ethanol for 3 minutes and the ascending aorta was harvested from the annulus to the origin of the brachiocephalic trunk. The harvested tissue was fixed in $70 \%$ ethanol for 24 hours at $4{ }^{\circ} \mathrm{C}$ and embedded in paraffin. Cross-sections $6-\mu \mathrm{m}$ thick were stained with orcein and Sirius red for elastin and collagen fibers, respectively. A monoclonal mouse antibody against alpha smooth muscle actin ( $\alpha$-SMA) (1A4 clone; DakoCytomation, Carpinteria Calif) was used and revealed by immunoperoxidase using a Vectastain Elite ABC kit (PK-6102; Vector Laboratory, Burlingame, Calif). Negative controls were generated by omission of the primary antibody, and a nonrelevant antibody.

Quantifications of internal vessel perimeter, medial thickness, medial area, and total wall area were realized on orcein stained aortas using AxioCamICc1 camera mounted on AxioImager D1 microscope with AxioVision software (Zeiss, Germany). For medial thickness a mean from four measurements taken at 3,6,9, and 12 o'clock was retained.

\section{Quantification of Gelatinase Activities}

After pulverization of the aortic tissue using a MultiSample Biopulverizer (BioSpec Products, VWR International, France), extracts were prepared with ice-cold $0.2 \%$ Triton $\mathrm{X}-100$ in $0.05 \mathrm{~mol} / \mathrm{L}$ Tris-HCl, $\mathrm{pH} 7.6$ containing $0.01 \mathrm{~mol} / \mathrm{L}$ calcium chloride. Protein content was determined by bicinchoninic acid assay quantification according to the manufacturer's recommendations. Fifteen microgram extracts per lane were subjected to a $10 \%$ sodium dodecyl sulfate polyacrylamide gel electrophoresis containing $0.1 \%$ gelatin. After electrophoresis, gels were washed in $2.5 \%$ Triton X-100 for 30 minutes, further incubated for 24 hours at room temperature in $50 \mathrm{mmol} / \mathrm{L}$ Tris, $\mathrm{pH} 8.2$ containing $10 \mathrm{mmol} / \mathrm{L}$ calcium chloride, and finally stained with $0.008 \%$ Coomassie brilliant blue (Sigma Chemical Co, St Louis, Mo). Quantitative analysis of matrix metalloproteinase (MMP) activity was performed using QuantityOne software (4.6.3, 2006; Bio-Rad, Hercules, Calif).

\section{Experimental Design}

Animals were randomly assigned to treatment groups before anesthesia. Fifteen rats received elastase and 6 received physiologic saline (control group). Rats were put to death at day 10 (5 elastase-treated and 3 controlgroup rats) and day 30 (10 elastase-treated and 3 control-group rats).

\section{Statistical Analysis}

Results are expressed as medians and range. Nonparametric tests were used for comparisons between groups: Mann-Whitney U test or Wilcoxon matched pairs test for comparison of 2 groups and Kruskal-Wallis test for comparison of 3 or more groups. All tests were performed using GraphPad Prism version 4 (2003; GraphPad Software, San Diego, Calif).

\section{RESULTS \\ Operative Observations}

Mortality was $19 \%$ (5 rats). Elastase application on the ascending aortic adventitia resulted in immediate and reproducible ascending aortic dilation (Figure 1). The dilation progressed during exposure to elastase. An aortic rupture occurred at the end of elastase application in 3 animals. One treated and 1 control animal died during follow-up, at day 2 and 3, respectively. The dilation was limited at the tubular portion of the ascending aorta without involvement of the aortic root. No aortic dilation was observed in control animals. Angiography performed before sacrifice 

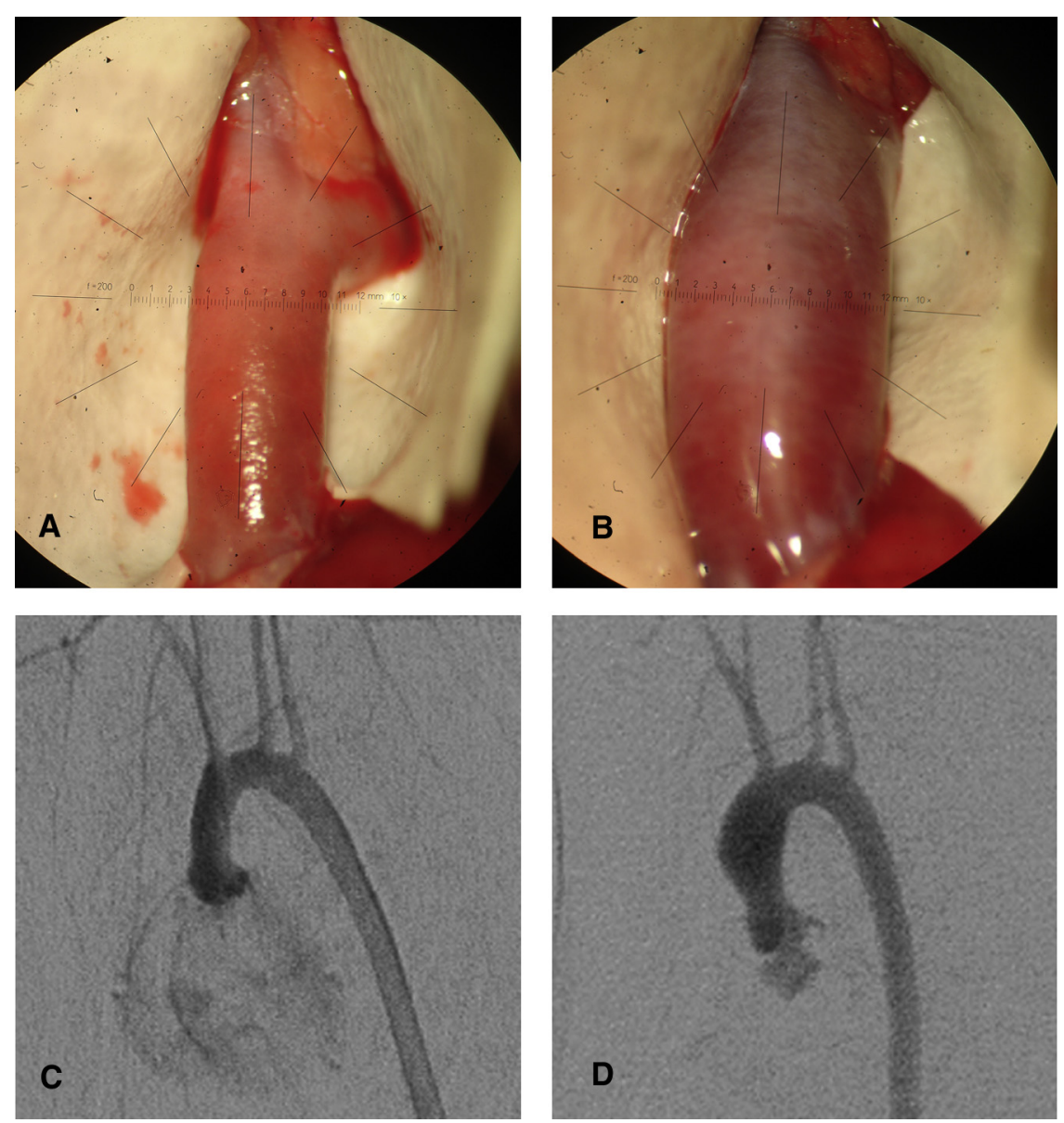

FIGURE 1. A, Intraoperative view through the operating microscope showing the entire ascending aorta with the beginning of the brachiocephalic trunk and aortic arch at the top, before elastase treatment. B, Ascending aorta after elastase treatment. C, Angiography performed at 30-day follow-up, before death, showing thoracic aorta in a control group animal and (D), a treated animal.

confirmed the presence of an aneurysm involving the tubular portion of the ascending aorta in elastase-treated rats (Figure 1).

\section{Aortic Diameters and Wall Thickness}

Iterative measurements by transthoracic ultrasound revealed a progressive and significant increase of the ascending aorta diameter in elastase-treated animals compared with saline-treated control animals. At 10- and 30-day follow-up median aortic diameter had an increase of $38 \%$ and $44 \%$, respectively, compared with the preoperative value $(P<.05$ for both) (See Figure 2 and Table 1$)$.

The medial layer of the treated aortas showed a significant thinning compared with medial thickness of control aortas (21\% and $26 \%$ decrease in medial thickness at $10-$ and 30-day follow-up, respectively; $P<.05$ ) (Figure $2, B$, and Table 1). However, the vascular wall layers are predicted to thin in response to vascular dilation alone (in the absence of any atrophic vessel wall changes); for example, in an aorta that increases from 3.4 to $4.9 \mathrm{~mm}$ in internal diameter, an initial hypothetical medial thickness of $131 \mu \mathrm{m}$ will decrease with $>31 \%$ to $90 \mu \mathrm{m}$ if the aortic wall mass remains unchanged. Standardization of the medial area measurements by dividing them to the corresponding internal perimeter of the vessel as proposed by Tang and colleagues ${ }^{12}$ showed no significant difference between elastase-treated and control aortas (Figure 2, C). This result indicates that the medial mass had not changed upon elastase application. Similarly, standardization of the total wall area (media + adventitia) measurements by dividing them to the internal perimeter of the vessel showed a significant increase in elastasetreated aortas compared with control aortas (Figure 2, D) that must be accounted for by an important increase in the adventitial area. These results indicate that the wall of the treated aortas increased in mass, predominantly in the adventitia, without significant medial thinning.

\section{Quantification of Gelatinases}

Pro MMP-2 levels demonstrated no change in elastasetreated aortas compared with controls at any time point. In contrast, active MMP-2 levels were not different from controls at 10 days but had increased significantly 30 days 


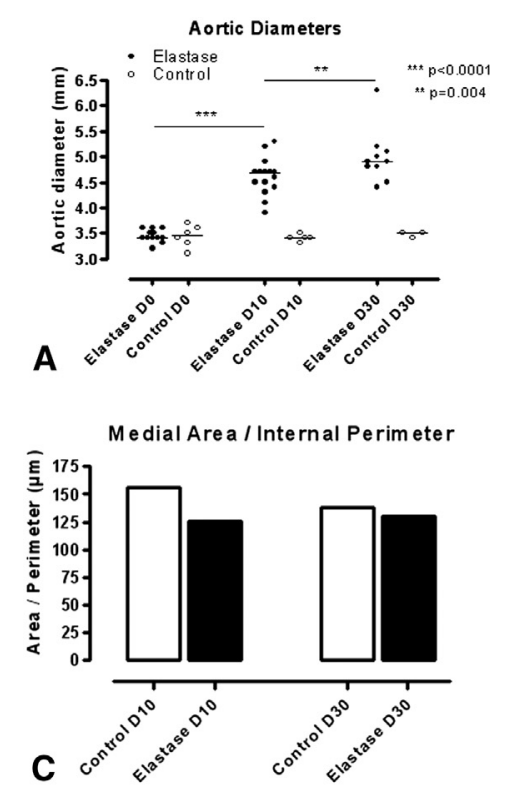

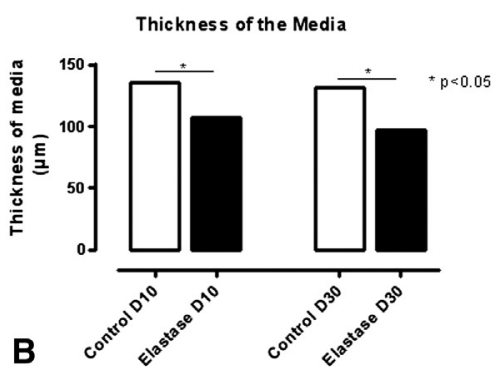

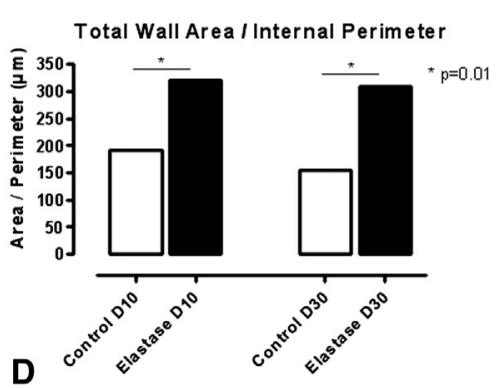

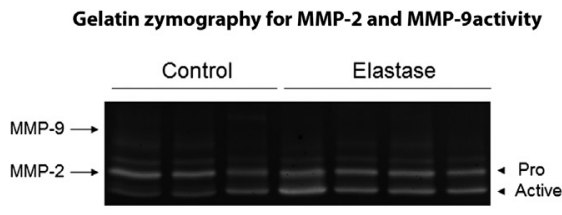

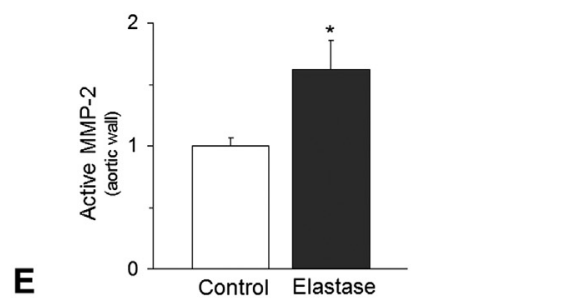

FIGURE 2. A, Ascending aortic diameters measured by transthoracic ultrasounds preoperatively (elastase $n=15$, control $n=6$ ) and at 10-day (elastase $\mathrm{n}=15$, control $\mathrm{n}=6$ ) and 30-day (elastase $\mathrm{n}=10$, control $\mathrm{n}=3$ ) follow-up. The graph shows each data point and median values (horizontal line). B, Thickness of the medial layer measured by computer-assisted videomicroscopy. The graph shows median values of each experimental group (elastase $\mathrm{n}=10$, control $\mathrm{n}=3$ ). C, Standardization of the area of the medial layer by dividing it to the corresponding internal perimeter of the vessel. The graph shows median values of each experimental group (elastase $n=10$, control $n=3$ ). D, Standardization of the total area of vessel wall by dividing it to the corresponding internal perimeter of the vessel. The graph shows median values of each experimental group (elastase $\mathrm{n}=10$, control $\mathrm{n}=3$ ). E, Activation of matrix metalloproteinase-2 (MMP-2) and matrix metalloproteinase-9 $(M M P-9)$ in the aortic wall of control $(\mathrm{n}=3)$ and elastase-treated $(\mathrm{n}=4)$ rats at 30 day follow-up. Representative zymogram is shown with a graph of quantitative analysis of active MMP-2 levels. Gelatin zymography detects 2 major bands corresponding to the size of proform and active form of MMP-2. $* P<.05$ versus control group.

after elastase treatment (Figure 2, E). Pro and active MMP-9 levels demonstrated no change in elastase-treated aortas compared with controls, at any studied time point.

\section{Histology}

Immediately after elastase treatment, the external part of the media exhibited extensive injury, with destruction of elastic lamellae on orcein staining (Figure 3,A), and disappearance of VSMCs as shown by anti $\alpha$-SMA immunostaining (Figure 3, B). The few remnant VSMCs in the inner aspect of the media had lost $\alpha$-SMA expression.
At day 10, there were fragmented and disarrayed elastic fibers in the media layer and the adventitia was intensely infiltrated with cells (Figure 3,C). Expression of $\alpha$-SMA remained faint in the media but had increased, compared with day 0 . Moreover, an intense $\alpha$-SMA immunostaining was observed in the adventitia (Figure 3,D). Sections from control animals showed normal aortic histology (Figure 3, $E$ and $F$ ).

At day 30, matrix deposition in the external part of the media and in the adventitia restored the thickness of the aortic wall (Figure 4). The limit between the severely injured

TABLE 1. Aortic diameters and aortic wall thicknesses measured by echography and microscopy, respectively

\begin{tabular}{|c|c|c|c|c|c|c|}
\hline \multirow[b]{2}{*}{ Measure } & \multicolumn{2}{|c|}{ Preoperative (day 0) } & \multicolumn{2}{|c|}{ Day 10} & \multicolumn{2}{|c|}{ Day 30} \\
\hline & $\begin{array}{l}\text { Control } \\
(n=6)\end{array}$ & $\begin{array}{l}\text { Elastase } \\
(\mathbf{n}=15)\end{array}$ & $\begin{array}{l}\text { Control } \\
(\mathbf{n}=\mathbf{3})\end{array}$ & $\begin{array}{c}\text { Elastase } \\
(n=5)\end{array}$ & $\begin{array}{l}\text { Control } \\
(\mathbf{n}=\mathbf{3})\end{array}$ & $\begin{array}{l}\text { Elastase } \\
(\mathbf{n}=10)\end{array}$ \\
\hline \multicolumn{7}{|l|}{ Echography measures } \\
\hline Diameter (mm) & $3.5(3.1-3.7)$ & $3.4(3.2-3.6)$ & $3.4(3.3-3.5)$ & $4.7 * * *(3.9-5.3)$ & $3.5(3.4-3.5)$ & $4.9 * *(4.4-6.2)$ \\
\hline \multicolumn{7}{|l|}{ Microscopy measures } \\
\hline Media thickness $(\mu \mathrm{m})$ & & & $135(85-255)$ & $107 *(25-264)$ & $131(96-194)$ & $97 *(26-219)$ \\
\hline $\begin{array}{l}\text { Media surface/ } \\
\text { perimeter }(\mu \mathrm{m})\end{array}$ & & & $156.3(136.7-167.2)$ & $125.4(97.5-14.3)$ & $137.8(121.4-156.8)$ & $130.0(107.3-183.1)$ \\
\hline $\begin{array}{c}\text { Total wall surface/ } \\
\text { perimeter }(\mu \mathrm{m})\end{array}$ & & & $192.1(172.1-217.2)$ & $319.9 *(302.1-339.2)$ & $155.7(144.4-179.9)$ & $308.9^{*}(228.8-370.2)$ \\
\hline
\end{tabular}

Data are presented as median (range). $* P<.05$ with respect to control value. $* * P=.004$ for the difference between day 10 and day 30 measures. $* * * P<.001$ for the difference between preoperative and day 10 measures. 

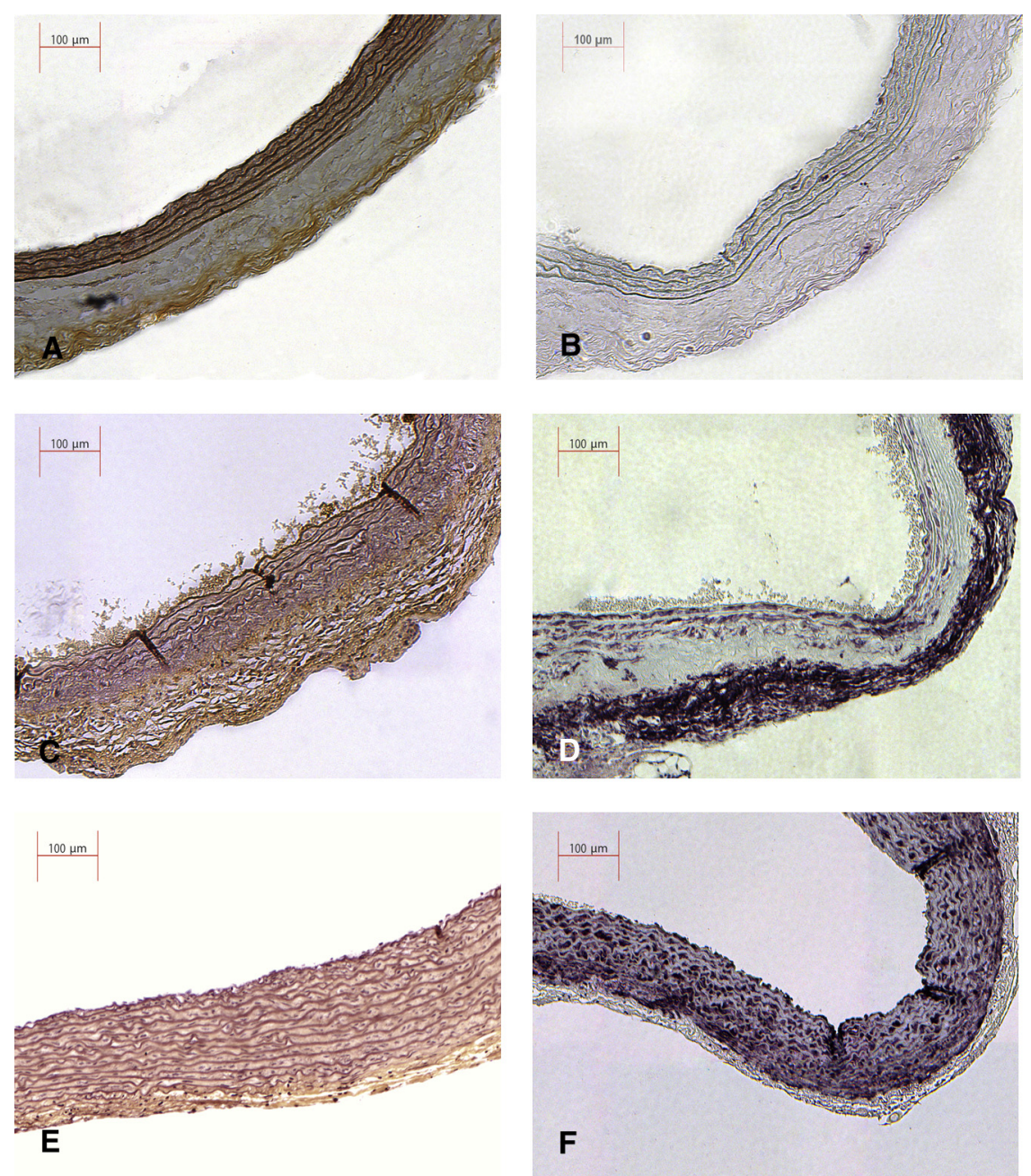

FIGURE 3. Histologic sections of treated (A-D) and control (E, F) ascending aortae stained with orcein for elastic fibers (A, C, and E) and $\alpha$-actin (B, D, and F). Immediately after elastase treatment, there was extensive destruction of elastic lamellae and no expression of $\alpha$-smooth muscle actin (A, B). At 10day follow-up, there were fragmented and disarrayed elastic fibers in the media and adventitia and faint expression of $\alpha$-smooth muscle actin in the media but an intense expression in the adventitia (C, D). Control aortae at 10-day follow-up show normal architecture of elastic lamellae and $\alpha$-smooth muscle actin expression (E, F).

outer part of the media and the inner part of the adventitia could not be distinguished. Orcein staining (Figure 4, B) showed persistent, marked elastic fiber fragmentation and disarray, resembling elastin injury in human ATAA. ${ }^{13}$ Sirius red staining showed less collaged fibers in the media but important collagen accumulation in the adventitia of elastasetreated aortas compared with controls (Figure 4, $C$ and $D$ ). Similarly, $\alpha$-SMA expression remained scarce in the media but was strong in the adventitia of treated aortas compared with controls (Figure 4, $E$ and $F$ ).

\section{DISCUSSION}

Our study demonstrates that elastase application onto the adventitia of the ascending aorta is feasible in rats through a sternotomy approach and generates ATAAs that expand in diameter as shown by transthoracic ultrasound measurement in vivo. We also demonstrate that although the media layer in experimental ATAA is thinned, there is no reduction of the medial area and that the crosssectional total area is increased, in comparison to nondilated, control ascending aorta. The VSMC decrease at the initial phase of ATAA formation is compensated for by the accumulation of $\alpha$-SMA positive cells in a disorganized extracellular matrix, a scheme specific to aneurysms of the ascending aorta, in sharp contrast to aneurysms in other locations of the aorta. ${ }^{1}$ Lastly, we show that activated MMP-2 contents are increased in experimental ATAA. Accordingly, adventitial application of elastase in rats is shown to result in ATAAs that share tissue and cellular organization common to that of ATAAs in human beings.

Previous studies have reported on the formation of experimental aneurysms at the level of the thoracic aorta. In 

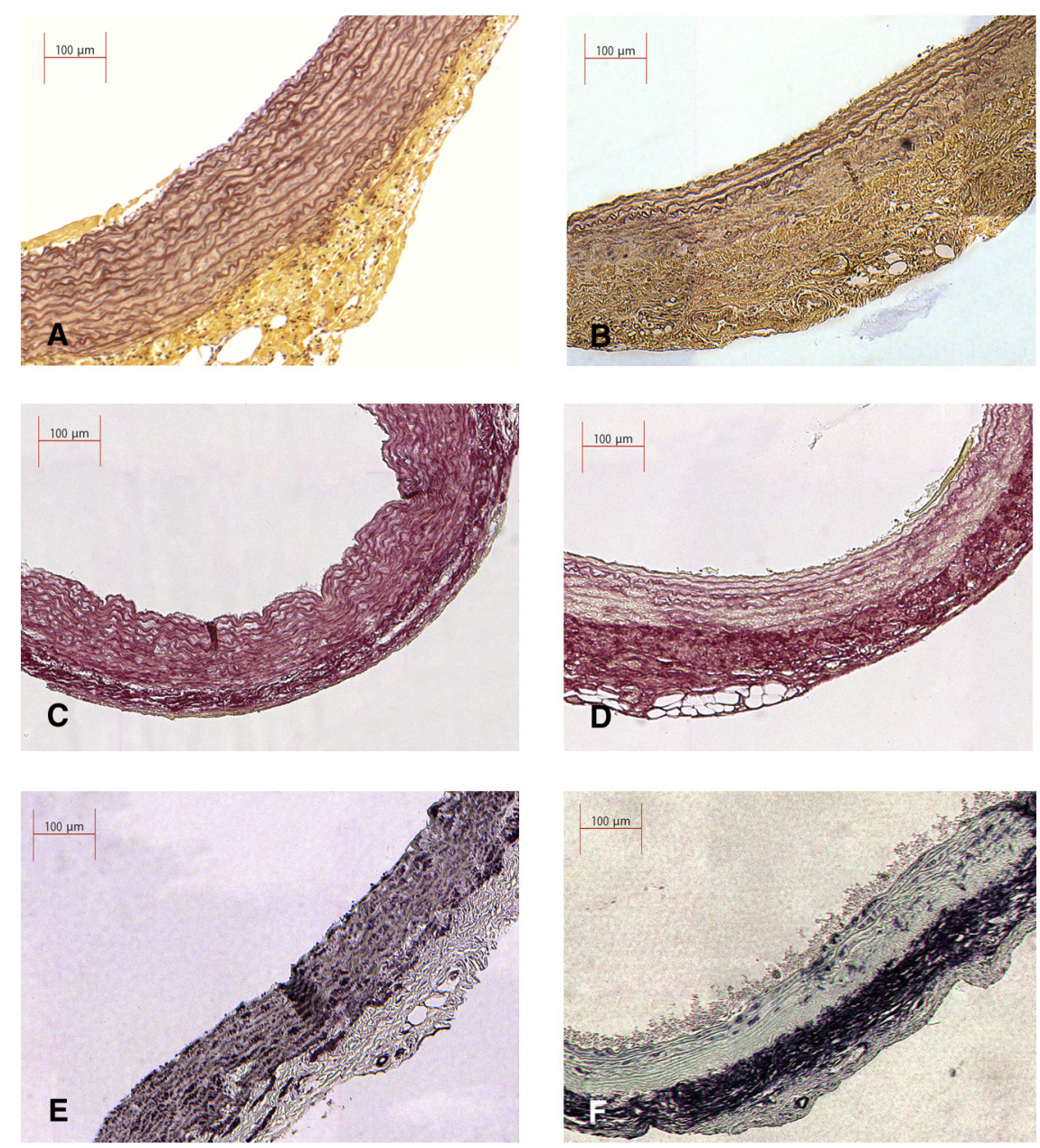

FIGURE 4. A, C, and E, Sections of control aortae at 30-day follow-up and (B, D, and F) treated aortae at 30-day follow-up. Elastase treatment produced marked elastic fiber fragmentation (orcein staining [A, B]), decreased collagen in the media but increased collagen production in the adventitia (Sirius red staining [C, D]). The loss of $\alpha$-smooth muscle actin expression persisted in the media of treated aortas and was accompanied by its expression in the adventitia $(\mathrm{E}, \mathrm{F})$.

blotchy mice, deficient in the elastin cross-linking lysyl oxidase, aneurysms were found involving both the ascending aorta and the arch, but also in other segments of the aorta. ${ }^{14}$ Expanding this founding observation, a recent model of both thoracic and abdominal aneurysms has been described, by infusing $\beta$-aminopropionitrile, a lysyl oxidase inhibitor, to mice with hypertension induced by angiotensin II or deoxycorticosterone acetate-salt. ${ }^{15}$ Ikonomidis and colleagues ${ }^{16}$ induced aneurysm formation in the distal descending thoracic aorta of mice by periadventitial application of calcium chloride, obtaining in this regionally distinct aortic segment a model sharing similarities with thoracoabdominal aneurysms in human beings, but different from degenerative ATAAs. ${ }^{16}$ None of these models were intended to induce reproducibly aneurysm formation limited to the ascending aorta, the predominant localization of thoracic aneurysms in human beings.

Other studies have described thoracic aneurysms in mice associated with monogenic defects to unravel the contribution of specific genes in syndromes involving the thoracic aorta in human beings, including Marfan syndrome and Loeys-Dietz syndrome. ${ }^{17}$ Other groups have obtained aneurysms of the ascending aorta and the aortic arch by overexpressing S100A12, a ligand to receptors for advanced glycation end products, targeted to VSMCs. ${ }^{18}$ These approaches are based on pathophysiologic assumptions at the molecular level, distinct from the most common form of ATAAs in human beings, referred to as degenerative. Here, we provide evidence that ATAAs can be generated independently of any genetic background, as in degenerative ATAAs in human beings.

Proteases have been shown to be determinant in aortic aneurysm formation. ${ }^{7}$ Previous reports have shown evidences of increased protease expression in ATAAs. ${ }^{10,19,20}$ Intraluminal infusion of elastase in rat abdominal aorta produces experimental aneurysms by medial elastolysis and secondary inflammatory response. ${ }^{21}$ This aneurysm model was used in numerous studies offering several 
insights on the mechanisms of abdominal aortic aneurysms development and permitted testing several new treatment approaches. ${ }^{22}$ However the anatomic and physiologic characteristics of the ascending aorta preclude intraluminal infusion. White and colleagues ${ }^{23}$ obtained infrarenal abdominal aneurysms in rabbits by elastase application onto the adventitia. Aortic diameters continued to increase up to 2 months of follow-up. Loss of elastin in the adventitia and the outer part of the media was observed.

Applying elastase on rat ascending aortic adventitia produced complete destruction of elastic lamellae and elastin fibers in the adventitia and external part of the media. There was significant thinning of the medial layer, but standardization of the medial area by dividing it to the internal perimeter of the vessel showed no difference with respect to controls, despite thinning of the media secondary to vascular dilation. Also there was a significant increase in total wall area in treated aortas compared with controls, due to an important increase in the adventitial area. Similar observations have been reported from human ATAAs. ${ }^{12}$

The histologic sections showed that most of the reparative process took place in the outer part of the media and in the adventitia. At 30-day follow-up we observed that these layers were intensely repopulated with $\alpha$-SMA-expressing cells that were probably responsible for the important collagen production shown by the Sirius red staining at the level of the outer media and adventitia. Although the thickness was restored by matrix deposition, the normal architecture of the vascular wall with regularly disposed elastic lamellae was not reestablished. Thus, histologic modifications induced by this approach appeared similar to our previous observations in patients operated on for ascending aortic aneurysms with tricuspid aortic valves. Indeed, at the level of maximal dilation we observed an important density of VSMCs but the typical lamellar organization of those cells was altered. ${ }^{13}$ Similar observations have been reported by other groups. ${ }^{12}$

The increased level of active MMP-2 observed at 30 days after elastase treatment might be responsible for the continuous increase in aortic diameters observed during the follow-up. Indeed MMP-2 has been associated with dedifferentiated smooth muscle cells that switched from a contractile to a synthetic phenotype, playing a role in aneurysmal expansion. ${ }^{20}$

Despite reproducing several observations from human pathology, this model has several limitations. Aneurysms obtained after elastase application onto the adventitia were limited to the tubular portion of the ascending aorta without involvement of the aortic root or the aortic valve, perhaps due to insufficient exposure of the aortic root to elastase. The ascending aorta has to be considered as 1 functional unit. Alteration of 1 of its components will have important consequences on the function of the aortic root as a whole. Thus, optimal aortic valve function (opening and closing) closely relies on aortic root morphology (eg, sinuses of Valsalva and sinotubular junction) and its elastic components. ${ }^{7}$ Conversely, primary or secondary aortic valve dysfunction resulting in significant stenosis or regurgitation will obviously lead to disturbed aortic blood flow patterns eventually contributing to the development or enhancement of aortic wall disease. ${ }^{24}$ However, ATAAs in human beings can develop without associated aortic valve incompetence.

\section{CONCLUSIONS}

We provide a description of the first model of degenerative ATAA in rodents, limited to the ascending aorta as the most common location of thoracic aortic aneurysms in human beings. Our finding that ATAAs obtained in rats after elastase application exhibit structural features common to human ATAAs suggests that the ascending aorta behaves in a particular and specific way to elastase injury. This new model may help provide insights into the pathophysiology of this common and life-threatening condition, allowing for the design of new therapeutic approaches.

\section{References}

1. Isselbacher EM. Thoracic and abdominal aortic aneurysms. Circulation. 2005; 111:816-28.

2. Olsson C, Thelin S, Stahle E, Ekbom A, Granath F. Thoracic aortic aneurysm and dissection: increasing prevalence and improved outcomes reported in a nationwide population-based study of more than 14,000 cases from 1987 to 2002 . Circulation. 2006;114:2611-8.

3. Michineau S, Dai J, Gervais M, Zidi M, Clowes AW, Becquemin JP, et al. Aortic length changes during abdominal aortic aneurysm formation, expansion and stabilisation in a rat model. Eur J Vasc Endovasc Surg. 2012;40:468-74.

4. Allaire E, Muscatelli-Groux B, Guinault AM, Pages C, Goussard A, Mandet C, et al. Vascular smooth muscle cell endovascular therapy stabilizes already developed aneurysms in a model of aortic injury elicited by inflammation and proteolysis. Ann Surg. 2004;239:417-27.

5. Dai J, Losy F, Guinault AM, Pages C, Anegon I, Degranges P, et al. Overexpression of transforming growth factor- $\beta 1$ stabilizes already-formed aortic aneurysms. Circulation. 2005;112:1008-15.

6. Dai J, Michineau S, Franck G, Desgranges P, Becquemin JP, Gervais M, et al. Long term stabilization of expanding aortic aneurysms by a short course of cyclosporine A through transforming growth factor-beta induction. PLoS One. 2011;6:e28903.

7. Radu NC, Gervais M, Allaire E, Loisance DY, Kirsch ME. Pathobiology of idiopathic ascending aortic aneurysms. Presse Med. 2009;38:1076-88.

8. LeLievre C, LeDouarin N. Mesenchymal derivatives of the neural crest: analysis of chimeric quail and chick embryos. J Embryol Exp Morphol. 1975;34:125-54.

9. Topouzis S, Majesky MW. Smooth muscle lineage diversity in the chick embryo. Two types of aortic smoth muscle cell differ in growth and receptor-mediated transcriptional responses to TGF $\beta$. Develop Biol. 1996;178:430-45.

10. Allaire E, Schneider F, Saucy F, Dai J, Cochennec F, Michineau S, et al. New insight in aetiopathogenesis of aortic diseases. Eur J Vasc Endovasc Surg. 2009;37: 531-7.

11. Radu CN, Michineau S, Hidalgo A, Blanc R, Gervais M, Loisance DY, et al. Validity of the sonographic measurement of the diameters of the ascending aorta in rats. Ultraschall Med. 2010;31:26-30.

12. Tang PCY, Coady MA, Lovoulos C, Dardik A, Aslan M, Elefteriades JA, et al Hyperplastic cellular remodeling of the media in ascending thoracic aortic aneurysm. Circulation. 2005;112:1098-105.

13. Kirsch EW, Radu NC, Gervais M, Allaire E, Loisance DY. Heterogeneity in the remodeling of aneurysms of the ascending aorta with tricuspid aortic valves. J Thorac Cardiovasc Surg. 2006;132:1010-6.

14. Andrews EJ, White WJ, Bullock LP. Spontaneous aortic aneurysms in blotchy mice. Am J Pathol. 1975;78:199-210. 
15. Kanematsu Y, Kanematsu M, Kurihara C, Tsou TL, Nuki Y, Liang EI, et al. Pharmacologically induced thoracic and abdominal aortic aneurysms in mice. Hypertension. 2010;55:1267-74.

16. Ikonomidis JS, Gibson WC, Gardner J, Sweterlitsch S, Thompson RP, Mukherjee R, et al. A murine model of thoracic aortic aneurysms. J Surg Res. 2003;115:157-63.

17. Dietz HC, Mecham RP. Mouse models of genetic diseases resulting from mutations in elastic fiber proteins. Matrix Biol. 2000;19:481-8.

18. Hofmann Bowman M, Wilk J, Heydemann A, Kim G, Rehman J, Lodato JA, et al S100A12 mediates aortic wall remodeling and aortic aneurysm. Circ Res. 2010; $106: 145-54$

19. Ikonomidis JS, Jones JA, Barbour JR, Stroud RE, Clark LL, Kaplan BS, et al Expression of matrix metalloproteinases and endogenous inhibitors within ascending aortic aneurysms of patients with bicuspid or tricuspid aortic valves. J Thorac Cardiovasc Surg. 2007;133:1028-36.

20. Lesauskaite V, Epistolato MC, Castagnini M, Urbonavicius S, Tanganelli P. Expression of matrix metalloproteinases, their tissue inhibitors, and osteopontin in the wall of thoracic and abdominal aortas with dilatative pathology. Hum Pathol. 2006;37:1076-84.

21. Anidjar S, Salzmann JL, Gentric D, Lagneau P, Camilleri JP, Michel JB. Elastaseinduced experimental aneurysms in rats. Circulation. 1990;82:973-81.

22. Dobrin PB. Animal models of aneurysms. Ann Vasc Surg. 1999;13:641-8.

23. White JV, Mazzacco SL. Formation and growth of aortic aneurysms induced by adventitial elastolysis. Ann N Y Acad Sci. 1996;800:97-120.

24. Robicsek F. Bicuspid versus tricuspid aortic valves. J Heart Valve Dis. 2003;12: 52-3 